\title{
ON THE ACCUMULATION OF THE ZEROS OF A BLASCHKE PRODUCT AT A BOUNDARY POINT
}

\author{
DAVID PROTAS
}

\begin{abstract}
Let $B$ be a Blaschke product with zeros $\left\{a_{n}\right\}$. The series $\sum\left(1-\left|a_{n}\right|^{2}\right) /\left|1-\bar{\zeta} a_{n}\right|^{\gamma}$, where $\gamma \geqq 1$ and $|\zeta|=1$, has been used by P. R. Ahern, D. N. Clark, G. T. Cargo, and others in the study of the boundary behavior of $B$ and various associated functions. In this paper the convergence of this series is shown to be equivalent to a condition on a reproducing kernel for a subspace of the Hardy space $H^{2}$. Some related conditions and corollaries are also given.
\end{abstract}

1. Introduction. Let $U$ denote the unit disc in the complex plane. For $0<\left|a_{n}\right|<1$ and $\sum\left(1-\left|a_{n}\right|\right)<\infty$, put

$$
B(z)=\prod_{n=1}^{\infty} \frac{\bar{a}_{n}}{\left|a_{n}\right|} \frac{a_{n}-z}{1-\bar{a}_{n} z} .
$$

$B$ is holomorphic in $U$ and is called a Blaschke product. If $\gamma \geqq 1$ and if $\zeta$ is any point on the boundary, $\partial U$, of $U$, we can associate with the sequence $\left\{a_{n}\right\}$, the series

$$
\sum_{n=1}^{\infty}\left(1-\left|a_{n}\right|^{2}\right) /\left|1-\bar{\zeta} a_{n}\right|^{\gamma}
$$

In [1] and [3], Ahern and Clark study properties necessary and sufficient for the convergence of (1) for integer values of $\gamma$. For general $\gamma$, Cargo [4] has given a necessary condition for the convergence of (1) in terms of the existence of certain tangential limits for $B$ and all its subproducts. This condition is shown by Linden and Somadasa in [7] to be not sufficient. A related condition, which is also necessary but not sufficient, can be found in [8]. In $\S 3$ of this paper, we give, for any $\gamma \geqq 1$, a necessary and sufficient condition for the convergence of (1). We also give the corresponding result for general inner functions.

Received by the editors June 10, 1971 and, in revised form, October 4, 1971 .

AMS 1970 subject classifications. Primary 30A76, 30A78; Secondary 30A31.

Key words and phrases. Blaschke product, Hardy space, inner function, invariant subspace, reproducing kernel.

(c) American Mathematical Society 1972 
In $\S 4$, a corollary is given which is motivated by some work on the segmental variation of Blaschke products by Cargo [5]. Also, we present another necessary and sufficient condition for the convergence of (1) when $\gamma$ is an integer. $\$ 5$ is devoted to an application, observed by Professor D. N. Clark, of the results of $\S 3$.

2. Preliminaries. For each $\zeta \in \partial U$, each $\gamma \geqq 1$, and each $m>0$, let $\Gamma=\Gamma_{\zeta, \gamma, m}$ be the "curve" in $U$ given by $\Gamma(\theta)=\left(1-m|\theta|^{\gamma}\right) e^{i \theta}$ for $0<|\theta|<$ $\min \left\{\pi, m^{-1 / i^{\prime}}\right\}$. $\Gamma$ encloses the region $R(m, \zeta, \gamma)$ which was introduced by Cargo in his study of tangential limits [4].

Let $H^{2}$ denote the usual Hardy class of functions holomorphic in $U$. A well-known theorem of Beurling states that any closed invariant subspace of $H^{2}$ is of the form $\phi H^{2}$, where $\phi$ is an inner function. That is, $\phi$ is a bounded holomorphic function in $U$ whose radial limits are of modulus one at almost all points of $\partial U$. If $\phi$ is an inner function, then $\phi=M B s$ where $M$ is a monomial, $B$ is a Blaschke product with zeros $\left\{a_{n}\right\}\left(a_{n} \neq 0\right)$, and $s$ is a singular inner function. By this we mean

$$
s(z)=\exp \left\{-\int_{0}^{2 \pi}\left(e^{i t}+z\right) /\left(e^{i t}-z\right) d \sigma(t)\right\}
$$

where $\sigma$ is a finite nonnegative singular measure. The function $s$, in turn, can be factored into the product of two singular inner functions, $s_{c}$ and $s_{a}$, corresponding to, respectively, a continuous measure $\sigma_{c}$ and a purely atomic measure $\sigma_{a}$ with $\sigma=\sigma_{c}+\sigma_{a}$. For a general discussion of the above see, for example, [9].

For any inner function $\phi$, we let $\left(\phi H^{2}\right)^{\perp}$ be the orthogonal complement of $\phi H^{2}$ in $H^{2}$. For each $z \in U$, define the function $K_{z}=K_{z}^{\phi}$ in $U$ by

$$
K_{z}(\lambda)=(1-\overline{\phi(z)} \phi(\lambda)) /(1-\bar{z} \lambda)
$$

It is observed in [1] and [2] that $K_{z} \in\left(\phi H^{2}\right)^{\perp}$ and that $\left(f, K_{z}\right)=f(z)$ for each $f \in\left(\phi H^{2}\right)^{\perp}$. In particular, we have

$$
\left\|K_{z}\right\|^{2}=K_{z}(z)=\left(1-|\phi(z)|^{2}\right) /\left(1-|z|^{2}\right),
$$

where $\left\|K_{z}\right\|$ stands for the $H^{2}$ norm of $K_{z}$.

3. The main result. We begin with the case of a Blaschke product.

THEOREM 1. Let $B$ be a Blaschke product with zeros $\left\{a_{n}\right\}$, and choose any $\zeta \in \partial U, \gamma \geqq 1$, and $m>0$. Then (1) converges if and only if

$$
\int_{\Gamma \zeta, \gamma_{, m}}\left\|K_{z}\right\|^{2}|d z|<\infty .
$$


Proof. In [1], it is shown that

$$
\left\|K_{z}\right\|^{2}=\sum_{n}\left|B_{n}(z)\right|^{2}\left(1-\left|a_{n}\right|^{2}\right) /\left|1-\bar{a}_{n} z\right|^{2},
$$

where $B_{n}$ is the subproduct of $B$ with zeros $a_{1}, \cdots, a_{n-1}$. The theorem is then obvious for any finite Blaschke product, and so we shall concern ourselves only with the case in which $B$ is an infinite Blaschke product. Also, we see by replacing $B$ with the Blaschke product with zeros $\left\{\bar{\zeta} a_{n}\right\}$ that we can assume that $\zeta=1$ without any loss in generality.

Now, let us assume that (1) converges. This implies, since $\zeta=1$, that $a_{n}$ can be real and positive for at most finitely many $n$. So, we can assume that $\arg a_{n}$ is never zero, where by $\arg a_{n}$ we mean the value of the argument of $a_{n}$ which is in the interval $(-\pi, \pi]$. Then, it is shown in [4] that the convergence of (1) implies that

$$
\sum_{n=1}^{\infty}\left(1-\left|a_{n}\right|\right) /\left|\arg a_{n}\right|^{\gamma}
$$

converges.

Let $c=\min \left\{\pi, m^{-1 / \gamma}\right\}$. We have, for $\Gamma=\Gamma_{1, \gamma, m}$,

$$
\int_{0}^{c}\left\|K_{\Gamma(\theta)}\right\|^{2} d \theta \leqq \sum_{n} 2\left(1-\left|a_{n}\right|\right) \int_{0}^{c}\left|1-\bar{a}_{n} \Gamma(\theta)\right|^{-2} d \theta .
$$

Set $t_{n}=\arg a_{n}$. By the law of cosines,

$$
\begin{aligned}
\left|1-\bar{a}_{n} \Gamma(\theta)\right|^{2} & =\left(1-\left|a_{n} \Gamma(\theta)\right|\right)^{2}+4\left|a_{n} \Gamma(\theta)\right| \sin ^{2} \frac{1}{2}\left(\theta-t_{n}\right) \\
& \geqq(1-|\Gamma(\theta)|)^{2}+\frac{1}{2}\left(\theta-t_{n}\right)^{2} \\
& =m^{2}|\theta|^{2 \gamma}+\frac{1}{2}\left(\theta-t_{n}\right)^{2}
\end{aligned}
$$

for $\theta$ close to 0 and any $n$ such that $t_{n}$ is close to 0 and $\left|a_{n}\right|>\frac{1}{2}$. So, there is a constant $c^{\prime}$ such that if $t_{n}$ is small and positive,

$$
\begin{aligned}
& \int_{0}^{c^{\prime}} \frac{d \theta}{\left|1-\bar{a}_{n} \Gamma(\theta)\right|^{2}} \\
& \leqq \int_{0}^{t_{n}-t_{n}^{\gamma} / 2} \frac{2 d \theta}{\left(\theta-t_{n}\right)^{2}}+\int_{t_{n}-t_{n}^{\gamma} / 2}^{t_{n}+t_{n}^{\gamma} / 2} \frac{d \theta}{m^{2} \theta^{2 \gamma}}+\int_{t_{n}+t_{n}^{\gamma} / 2}^{c^{\prime}} \frac{2 d \theta}{\left(\theta-t_{n}\right)^{2}} \\
&= 4 t_{n}^{-\gamma}-2 t_{n}^{-1}+\left[\left(t_{n}-t_{n}^{\gamma} / 2\right)^{1-2 \gamma}-\left(t_{n}+t_{n}^{\gamma} / 2\right)^{1-2 \gamma}\right] /(2 \gamma-1) m^{2} \\
& \quad+4 t_{n}^{-\gamma}-2 /\left(c^{\prime}-t_{n}\right) \\
&= O\left(1 / t_{n}^{\gamma}\right) \quad\left(t_{n} \rightarrow 0\right),
\end{aligned}
$$

where the estimate on $\left[\left(t_{n}-t_{n}^{\gamma} / 2\right)^{1-2 \gamma}-\left(t_{n}+t_{n}^{\gamma} / 2\right)^{1-2{ }^{\prime \prime}}\right]$ can be made by using its binomial expansion. If $t_{n}$ is close to 0 and negative, we note that $\left|1-a_{n} \Gamma(\theta)\right|<\left|1-\bar{a}_{n} \Gamma(\theta)\right|$ for $\theta>0$ and apply the above to $\left|1-a_{n} \Gamma(\theta)\right|$. 
It then follows from the convergence of (3) that $\int_{0}^{c}\left\|K_{\Gamma(\theta)}\right\|^{2} d \theta<\infty$. The finiteness of the integral over the interval $(-c, 0)$ is derived analogously. Then (2) follows since $\left|\Gamma^{\prime}(\theta)\right|$ is bounded for $-c<\theta<c$.

Now for the converse, assume that (2) holds. It is easily seen that

$$
B^{\prime}(z)=\sum_{n=1}^{\infty} \widetilde{B}_{n}(z)\left(1-\left|a_{n}\right|^{2}\right) /\left(1-\bar{a}_{n} z\right)^{2},
$$

where $\widetilde{B}_{n}(z)=B(z)\left(1-\bar{a}_{n} z\right) /\left(z-a_{n}\right)$. Then, $\left\|K_{z}\right\|^{2} \geqq|B(z)| \cdot\left|B^{\prime}(z)\right|=\frac{1}{2}\left|D^{\prime}(z)\right|$, where $D=B^{2}$. So, (2) implies that $\int_{\Gamma}\left|D^{\prime}(z)\right||d z|<\infty$. Thus, the Blaschke product $D$ has a limit as $z \rightarrow 1$ along $\Gamma$. Also, (2) implies that

$$
\int_{\Gamma}(1-|D(z)|) /(1-|z|)|d z|<\infty .
$$

Then if $c=\min \left\{\pi, m^{-1 / \gamma}\right\}, \int_{-c}^{c}(1-|D(\Gamma(\theta))|)|\theta|^{-\gamma} d \theta<\infty$ since $\left|\Gamma^{\prime}(\theta)\right|$ is bounded away from 0 . So, $\sup \{|D(z)|: z \in \Gamma\}=1$, and $|D(z)| \rightarrow 1$ as $z \rightarrow 1$ along $\Gamma$. Thus, $|B(z)| \rightarrow 1$ as $z \rightarrow 1$ along $\Gamma$.

It now follows from (2) and the series expansion for $\left\|K_{z}\right\|$ that

$$
\int_{-c}^{c} \sum_{n}\left(1-\left|a_{n}\right|^{2}\right) /\left|1-\bar{a}_{n} \Gamma(\theta)\right|^{2} d \theta<\infty,
$$

if we again use the fact that $\left|\Gamma^{\prime}(\theta)\right|$ is bounded away from 0 . This implies that

$$
\sum_{n}\left(1-\left|a_{n}\right|^{2}\right) \int_{I}\left|1-\bar{a}_{n} \Gamma(\theta)\right|^{-2} d \theta<\infty,
$$

where $t_{n}=\arg a_{n}, m^{\prime}=\min \left\{m, \frac{1}{2}\right\}$, and $I=\left(t_{n}-m^{\prime}\left|t_{n}\right|^{\gamma}, t_{n}\right)$ if $t_{n}>0$ and $I=\left(t_{n}, t_{n}+m^{\prime}\left|t_{n}\right|^{\gamma}\right)$ if $t_{n}<0$.

Since $B(z)$ has a limit of modulus one as $z \rightarrow 1$ along $\Gamma$, a theorem of Lindelöf (see [6, p. 460]) says that $B(z)$ has a limit of modulus one as $z \rightarrow 1$ inside $\Gamma$. So, there is an integer $n_{0}$ such that $a_{n}$ is outside of $\Gamma$ for each $n \geqq n_{0}$. In other words, $1-\left|a_{n}\right|<m\left|\arg a_{n}\right|^{\gamma}$ if $n \geqq n_{0}$. Then,

$$
\begin{aligned}
\left|1-\bar{a}_{n} \Gamma(\theta)\right|^{2} & =\left(1-\left|a_{n} \Gamma(\theta)\right|\right)^{2}+4\left|a_{n} \Gamma(\theta)\right| \sin ^{2} \frac{1}{2}\left(t_{n}-\theta\right) \\
& \leqq\left(1-\left|a_{n}\right|+m|\theta|^{\gamma}\right)^{2}+\left(t_{n}-\theta\right)^{2} \\
& \leqq\left(m\left|t_{n}\right|^{\gamma}+m|\theta|^{\gamma}\right)^{2}+\left(t_{n}-\theta\right)^{2} \leqq 5 m^{2}\left|t_{n}\right|^{2 \gamma}
\end{aligned}
$$

if $\theta \in I$ and $n \geqq n_{0}$. So,

$$
\sum_{n=n_{0}}^{\infty}\left(1-\left|a_{n}\right|^{2}\right) /\left|\arg a_{n}\right|^{\gamma}<\infty
$$

The convergence of (1) follows since $\sin \left|\arg a_{n}\right| \leqq\left|1-a_{n}\right|$. 
Next let us extend Theorem 1 to the case of a general inner function. Since there is much duplication with the proof of Theorem l, we shall only sketch the proof of the extension.

THEOREM 2. Let $\phi=M B s$ be an inner function, and choose any $\zeta \in \partial U$, $\gamma \geqq 1$, and $m>0$. Then (1) converges and

$$
\int_{0}^{2 \pi}\left|1-\bar{\zeta} e^{i t}\right|^{-\gamma} d \sigma(t)
$$

converges if and only if

$$
\int_{\Gamma_{\zeta, \gamma, m}}\left\|K_{z}^{\phi}\right\|^{2}|d z|<\infty .
$$

Proof. As before, we can assume that $\zeta=1$. Also, we shall assume, in order to avoid trivialities, that $B$ is an infinite Blaschke product and $s$ has infinitely many point masses and a nontrivial continuous part. Let us note that if $M(z)=b z^{k},\left\|K_{z}^{M}\right\|^{2}=\left(1-|z|^{2 k}\right) /\left(1-|z|^{2}\right)$ since $|b|=1$. So, (5) with $\phi$ replaced by $M$ always holds.

Now let us assume that (1) and (4) converge. Then by Theorem 1, (5) with $\phi$ replaced by $B$ holds. Since (4) converges, (4) with $\sigma$ replaced by $\sigma_{c}$ converges. In [2], it is shown that

$$
\left\|K_{z}^{\phi_{c}}\right\|^{2}=2 \int_{0}^{\pi}\left|s_{c, t}(z)\right|^{2} /\left|1-e^{-i t} z\right|^{2} d \sigma_{c}(t),
$$

where $s_{c, t}$ is the inner function given by

$$
s_{c, t}(z)=\exp \left\{-\int_{0}^{t}\left(e^{i \theta}+z\right) /\left(e^{i \theta}-z\right) d \sigma_{c}(\theta)\right\} .
$$

The proof that (5) with $\phi$ replaced by $s_{c}$ holds now can be seen to parallel the corresponding part of the proof of Theorem 1. Also in [2], there is a series representation for $\left\|K_{z}^{\phi_{a}}\right\|$, which we can use to prove that (5) with $\phi$ replaced by $s_{a}$ holds.

If $\phi$ and $\psi$ are any two inner functions, then

and so,

$$
1-|\phi(z) \psi(z)|^{2}=1-|\phi(z)|^{2}+|\phi(z)|^{2}\left(1-|\psi(z)|^{2}\right),
$$

$$
\left\|K_{z}^{\phi \psi}\right\|^{2}=\left\|K_{z}^{\phi}\right\|^{2}+|\phi(z)|^{2}\left\|K_{z}^{\psi}\right\|^{2} \leqq\left\|K_{z}^{\phi}\right\|^{2}+\left\|K_{z}^{\psi}\right\|^{2} .
$$

Therefore, the results of the last paragraph can be combined to show that (5) holds.

Conversely, assume that (5) holds. If $\phi$ and $\psi$ are any two inner functions, $1-|\phi(z) \psi(z)|^{2} \geqq 1-|\phi(z)|^{2}$, and so $\left\|K_{z}^{\phi \psi}\right\|^{2} \geqq\left\|K_{z}^{\phi}\right\|^{2}$. Thus, (5) holds 
with $\phi$ replaced by $B, s_{c}$, or $s_{a}$. Therefore, (1) converges, and in an analogous way (4) with $\sigma$ replaced by $\sigma_{c}$ or $\sigma_{a}$ converges.

4. The variation of an inner function. In [5], Cargo proves that the convergence of (1) with $\gamma=1$ implies the finiteness of the length of the curve which is the image under $B$ of the line segment joining any point $a \in U$ with $\zeta$. This leads us to

COROllary. Let $\phi=M B$ s be an inner function, and choose any $\zeta \in \partial U$, $\gamma \geqq 1$, and $m>0$. If (1) and (4) converge, then

$$
\int_{\Gamma_{\zeta, \gamma, m}}\left|\phi^{\prime}(z)\right||d z|<\infty .
$$

Proof. As we noted in the proof of Theorem $1,\left\|K_{z}^{B}\right\|^{2} \geqq|B(z)| \cdot\left|B^{\prime}(z)\right|$. It is shown in [4] that the convergence of (1) implies that $|B(z)| \rightarrow 1$ as $z \rightarrow \zeta$ along $\Gamma$. So, (6) with $\phi$ replaced by $B$ follows from (2). In a similar way, we can show that (6) also holds with $\phi$ replaced by $s_{c}$ or $s_{a}$. The desired result then follows directly.

In [3], Ahern and Clark prove that (1) converges for an integer value of $\gamma$ if and only if the $(\gamma-1)$ st derivative of each subproduct of $B$, including $B$ itself, has a finite radial limit at $\zeta$. This proves half of

THEOREM 3. Let $B$ be a Blaschke product with zeros $\left\{a_{n}\right\}$, and choose any $\zeta \in \partial U$ and any positive integer $\gamma$. Then (1) converges if and only if for each subproduct $f$ of $B$, including $B$ itself, we have

$$
\int_{0}^{1}\left|f^{(\gamma)}(\zeta r)\right| d r<\infty
$$

Proof. If (7) holds for each subproduct $f$ of $B$, we can use the just mentioned result of Ahern and Clark to immediately give the convergence of (1). So, let us now assume that (1) converges. As usual we can take $\zeta$ to be 1 . So we can assume that (3) converges.

In [1], it is noted that

$$
B^{(\gamma)}(r)=\sum_{j=0}^{\gamma-1}\left(\begin{array}{c}
\gamma-1 \\
j
\end{array}\right) \sum_{n=1}^{\infty} \widetilde{B}_{n}^{(\gamma-1-j)}(r) \frac{(j+1) ! \bar{a}_{n}^{j}\left(1-\left|a_{n}\right|^{2}\right)}{\left(1-\bar{a}_{n} r\right)^{j+2}}
$$

Also from [1], we see that we can find a constant $C_{0}$ such that $\left|\tilde{B}_{n}^{(\gamma-1-j)}(r)\right| \leqq C_{0}$ for any $0<r<1, j=0,1, \cdots, \gamma-1$, and $n=1,2, \cdots$. Then

$$
\int_{0}^{1}\left|B^{(\gamma)}(r)\right| d r \leqq C_{1} \sum_{n=1}^{\infty}\left(1-\left|a_{n}\right|^{2}\right) \int_{0}^{1}\left|1-\bar{a}_{n} r\right|^{-(\gamma+1)} d r
$$


for some constant $C_{1}$. By the law of cosines, $\left|1-\bar{a}_{n} r\right|^{2} \geqq(1-r)^{2}+\frac{1}{4}\left|t_{n}\right|^{2}$ for $t_{n}=\arg a_{n}$ close to 0 . Then for any such $n,\left|1-\bar{a}_{n} r\right|^{\gamma+1} \geqq(1-r)^{\gamma+1}+$ $k^{-1}\left|t_{n}\right|^{\gamma+1}, k=2^{\gamma+1}$. So,

$$
\begin{aligned}
\int_{0}^{1}\left|1-\bar{a}_{n} r\right|^{-(\gamma+1)} d r & \leqq \int_{0}^{1-\left|t_{n}\right|}(1-r)^{-(\gamma+1)} d r+\int_{1-\left|t_{n}\right|}^{1} k\left|t_{n}\right|^{-(\gamma+1)} d r \\
& =(k+1 / \gamma)\left|t_{n}\right|^{-\gamma}-1 / \gamma .
\end{aligned}
$$

Therefore, the convergence of (3) implies that (7) holds for $f=B$. If $f$ is any other subproduct of $B$, the zeros of $f$ will satisfy $\sum\left(1-\left|a_{n}\right|^{2}\right) /\left|1-a_{n}\right|^{\gamma}<\infty$, and so we can apply the above work directly to $f$ to show that (7) holds for $f$.

It is possible to extend Theorem 3 to general inner functions by introducing the concept of divisor as was done in [3].

5. An application. Let $\phi$ be any inner function, and let $\mu$ be any complex number of modulus less than 1 . It is easily checked that $\phi_{\mu}(z)=$ $(\phi(z)-\mu) /(1-\bar{\mu} \phi(z))$ is again an inner function.

THEOREM 4. Let $\phi=M B$ s be an inner function, and let $\mu$ be any complex number of modulus less than 1 . If, for any $\zeta \in \partial U$ and any $\gamma \geqq 1,(1)$ and (4) converge, then the corresponding sum and integral for $\phi_{\mu}$ also converge.

Proof. Let us compute

$$
\begin{aligned}
\left\|K_{z}^{\phi_{\mu}}\right\|^{2} & =\left(1-|\phi(z)-\mu|^{2}|1-\bar{\mu} \phi(z)|^{-2}\right) /\left(1-|z|^{2}\right) \\
& =|1-\bar{\mu} \phi(z)|^{-2}\left(|1-\bar{\mu} \phi(z)|^{2}-|\phi(z)-\mu|^{2}\right) /\left(1-|z|^{2}\right) \\
& =|1-\bar{\mu} \phi(z)|^{-2}\left(\left(1-|\mu|^{2}\right)-\left(1-|\mu|^{2}\right)|\phi(z)|^{2}\right) /\left(1-|z|^{2}\right) \\
& =\left(1-|\mu|^{2}\right)|1-\bar{\mu} \phi(z)|^{-2}\left\|K_{z}^{\phi}\right\|^{2} .
\end{aligned}
$$

Since (1) and (4) converge for $\phi$, Theorem 2 implies that (5) holds for $\phi$. But since $|\mu|<1$, the above computation implies that (5) holds for $\phi_{\mu}$. Again applying Theorem 2, we see that (1) and (4) converge for $\phi_{\mu}$.

The above theorem gives us information about the $\mu$ points of a Blaschke product $B$. Let $\left\{b_{n}\right\}$ be the sequence of all points (counting multiplicity) for which $B\left(b_{n}\right)=\mu$. Choose any $\zeta \in \partial U$ and any $\gamma \geqq 1$. If (1) converges, then (1) with the sequence $\left\{a_{n}\right\}$ replaced by the sequence $\left\{b_{n}\right\}$ converges by Theorem 4 since $\left\{b_{n}\right\}$ is the sequence of zeros of $B_{\mu}$.

\section{REFERENCES}

1. P. R. Ahern and D. N. Clark, Radial limits and invariant subspaces, Amer. J. Math. 92 (1970), 332-342. MR 41 \#7117.

2. - On functions orthogonal to invariant subspaces, Acta Math. 124 (1970), 191-204. MR 41 \#8981a. 
3. P. R. Ahern and D. N. Clark, Radial Nth derivatives of Blaschke products, Math. Scand. 28 (1971), 189-201.

4. G. T. Cargo, Angular and tangential limits of Blaschke products and their successive derivatives, Canad. J. Math. 14 (1962), 334-348. MR 25 \#204.

5. - The segmental variation of Blaschke products, Duke Math. J. 30 (1963), 143-149. MR 26 \#2616.

6. P. Dienes, The Taylor series, Clarendon Press, Oxford, 1931.

7. C. N. Linden and H. Somadasa, On tangential limits of Blaschke products, Arch. Math. (Basel) 18 (1967), 416-424. MR 38 \#2306.

8. D. Protas, Tangential limits of functions orthogonal to invariant subspaces, Trans. Amer. Math. Soc. (to appear).

9. W. Rudin, Real and complex analysis, McGraw-Hill, New York, 1966. MR 35 $\# 1420$.

Department of Mathematics, San Fernando Valley State College, NorthRIDGe, CALIFORNIA 91324 